\title{
Recuperación de la artroplastia de rodilla a través de la movilización pasiva continua
}

\author{
Recovery from total knee arthroplasty through \\ continuous passive motion
}

\author{
B. Sánchez Mayo', J. Rodríguez-Mansilla², B. González Sánchez²
}

\section{RESUMEN}

El propósito de este estudio es conocer los efectos de la movilización pasiva continua en pacientes intervenidos de artroplastia total de rodilla. Se desarrolló una estrategia de búsqueda en las bases de datos CSIC, PubMed, Cochrane Library Plus, Dialnet y PEDro con el fin de captar todos aquellos ensayos clínicos, en inglés y español, indexados de enero del 2000 a noviembre del 2014. Se identificaron 537 publicaciones, pero se incluyeron 12 estudios en esta revisión. La valoración de 1.153 pacientes demuestra que no existe una diferencia significativa en la mejora de la amplitud articular, dolor, equilibrio, marcha, cicatrización y estancia hospitalaria utilizando la movilización pasiva continua frente al tratamiento de fisioterapia habitual para la artroplastia total de rodilla. La aplicación de la movilización pasiva continua, a largo plazo, no aporta ningún beneficio en cuanto a la amplitud del rango articular, dolor y mejora de bipedestación y marcha en comparación con el tratamiento convencional de fisioterapia postoperatorio en la artroplastia total de rodilla. A corto plazo se consigue una mejora del rango de movilidad articular a la flexión de la rodilla.

Palabras clave: Movilización pasiva continua: Artroplastia. Gonartrosis. Rehabilitación.

\begin{abstract}
The purpose of this study was to know the effects of continuous passive mobilization in patients who underwent total knee arthroplasty. A search strategy was developed to retrieve all clinical trials, written in English and/or Spanish, published in the electronic search databases PubMed, Cochrane Library Plus, Dialnet, CSIC and PEDro. The inclusion criteria were: clinical trials published from January 2000 until November 2014 in English or Spanish. Out of 537 clinical trials that were potentially relevant, a total of 12 were included in this review. The evaluation of 1,153 patients shows that there is no significant difference in improving the range of the joint, pain, balance, motion, healing and hospital stay using continuous passive mobilization against the regular physiotherapy treatment for total knee arthroplasty. The application of continuous passive mobilization in the long-term does not provide any benefit in terms of the breadth of the range of the joint, pain and improvement of standing and motion in comparison with conventional postoperative physiotherapy treatment in total knee arthroplasty. In the short term an improvement is obtained in the range of joint motion in knee flexion.
\end{abstract}

Keywords. Continuous passive motion. Arthroplasty. Gonarthrosis. Rehabilitation.
1. Facultad de Medicina. Universidad de Extremadura. Badajoz. España.

2. Departamento Terapéutico Médico-Quirúrgica. Facultad de Medicina. Universidad de Extremadura. Badajoz. España.

Recepción: 22 de enero de 2015

Aceptación provisional: 23 de marzo de 2015

Aceptación definitiva: 13 de abril de 2015

\section{Correspondencia:}

Juan Rodríguez Mansilla

Departamento Terapéutica Médico-Quirúrgica.

Facultad de Medicina.

Universidad de Extremadura.

Avda. de Elvas S/N

06006 Badajoz

Email: jrodman@unex.es 


\section{INTRODUCCIÓN}

El envejecimiento genera una serie de cambios en el aparato osteoarticular derivados del paso del tiempo (cambios fisiológicos), de las secuelas que dejan las enfermedades, de los traumatismos acumulados a lo largo de la vida (cambios patológicos) e, incluso, del propio tipo de vida que haya desarrollado cada individuo. Estos cambios, unidos a los que tienen lugar en otros sistemas vinculados al aparato locomotor (músculos, pérdidas visuales o auditivas, etc.), generan alteraciones biomecánicas en los huesos y las articulaciones, favoreciendo la aparición progresiva de procesos patológicos de tipo degenerativo o articular ${ }^{1}$.

Dentro de estos procesos patológicos, la artrosis es el más frecuente y es la causa más habitual de incapacidad ${ }^{2}$ generando importantes implicaciones sanitarias, sociales, económicas y políticas ${ }^{3}$

Según indica la evidencia médica ${ }^{2,4}$, la artrosis aparece a partir de los 50 años, aumentando rápidamente con la edad y siendo más frecuente en las mujeres. Aunque afecta a las articulaciones de la columna y de las manos, gran parte de su importancia deriva de su localización en las rodillas y caderas, articulaciones esenciales para el desplazamiento y la deambulación ${ }^{5}$, lo cual tiene una gran repercusión en la calidad de vida.

La artrosis es una artropatía degenerativa que se produce al alterarse las propiedades mecánicas del cartílago y del hueso subcondral. Puede afectar a una sola articulación, siendo más habitual que se afecten varias a la vez

Los principales síntomas de esta enfermedad son: dolor articular, rigidez de menos de 30 minutos de duración, limitación de la movilidad, crepitación, inestabilidad articular, incapacidad funcional, derrame ocasional y grados variables de inflamación local ${ }^{6}$.

La literatura médica señala que el tratamiento de la artrosis pasa por diferentes fases $^{7}$. En un principio, generalmente, se realiza un tratamiento no farmacológico (educación, ejercicios, pérdida de peso, calzado adecuado) como medida de prevención de la misma. Según la evolución de la enfermedad se añade fisioterapia y analgésicos simples para aliviar el dolor. A medida que la enfermedad va progresando se incluirá un tratamiento farmacológico con AINE y opioides principalmente. Finalmente, cuando la enfermedad está muy avanzada, presenta gravedad de los síntomas y las medidas anteriores no han sido eficaces, se lleva a cabo tratamiento el quirúrgico ${ }^{8}$.

La intervención consiste en una corrección quirúrgica de las desviaciones del eje articular y el recambio articular de la rodilla por una articulación artificial ${ }^{8}$. A este tipo de intervención se le denomina artroplastia. Las investigaciones demuestran ${ }^{2,5,8}$ que una de las intervenciones más habituales y que en los últimos años se ha incrementado es la artroplastia total de rodilla ${ }^{2}$, cuyo propósito principal es aliviar el dolor e intentar mejorar la función articular ${ }^{8}$ de aquellas personas que presentan gonartrosis y en las cuales los tratamientos conservadores no han dado resultado.

Algunos investigadores afirman que, tras la colocación de la prótesis, es importante la realización de una rehabilitación adecuada para que el proceso de recuperación resulte exitoso $^{2}$. Esta rehabilitación se basa, principalmente, en la actuación de la fisioterapia cuyos objetivos son remitir los síntomas que presentan y mejorar las carencias funcionales postoperatorias. Para ello, esta disciplina se vale de una serie de ejercicios específicos que mejorarán el rango de movimiento, fortalecimiento, estiramiento muscular, y movilización de tejidos blandos?

En el periodo postoperatorio, para el trabajo articular, en muchas ocasiones se utiliza la movilización pasiva continua. Se trata de una técnica de rehabilitación que consiste en mover la articulación afectada en el rango de movilidad natural con una máquina diseñada para ese fin: artromotor. Su principio de aplicación es la oscilación sinusoidal de la presión intraarticular que hace que se acelere el drenaje de la hemartrosis, previniendo la acumulación extensa de edema en los tejidos periarticulares, lo que favorece la prevención de la rigidez articular'.

El artromotor es un dispositivo electromecánico alimentado por un motor 
eléctrico capaz de movilizar una articulación en uno u otro sentido. Este tipo de aparatos deben permitir la regulación de la fuerza movilizadora, la velocidad del movimiento, el tiempo de reposo, el número de repeticiones y el límite de la amplitud articular. Uno de los más utilizados es el artromotor de rodilla que moviliza la articulación alternativamente en flexión y extensión ${ }^{10}$.

Esta técnica fue utilizada por primera vez en 1980 siendo en 1982 cuando se determinaron inicialmente los beneficios de la misma en la rehabilitación de la rodilla tras la artroplastia total: menor estancia hospitalaria, menor número de movilizaciones forzadas, mayor rango de movilidad articular a los 10 días y menor requerimiento analgésico. Desde entonces se popularizó el uso de la movilización pasiva continua en los servicios de rehabilitación tras la cirugía de la rodilla ${ }^{11}$.

Por todo lo anteriormente expuesto, esta revisión sistemática cualitativa tiene por objetivo analizar los efectos de la movilización pasiva continua en pacientes intervenidos de artroplastia total de rodilla.

\section{METODOLOGÍA}

Revisión sistemática cualitativa de ensayos clínicos controlados. Se admitieron aquellos artículos que estuvieran publicados en inglés o en español, y cuya fecha de publicación se encontrase ubicada entre enero de 2000 y noviembre de 2014.

Todos los estudios tenían que estar dirigidos a pacientes que tuvieran programada una operación de prótesis total de rodilla, independientemente de que esta intervención fuese unilateral o bilateral, y que fuesen a recibir el tratamiento rehabilitador postoperatorio mediante fisioterapia y/o movilización pasiva continua.

Se excluyeron todos aquellos artículos científicos que tratasen otras enfermedades o complicaciones secundarias a la intervención, aquellos artículos en los que no se utilizaba ni la movilización pasiva continua ni la fisioterapia o que utilizaban técnicas nuevas de tratamiento en comparación con el tratamiento de fisioterapia tras la artroplastia total de rodilla, y todos los no encuadrados en la tipología de ensayos clínicos controlados.

\section{Estrategia de búsqueda}

Para la realización de la búsqueda bibliográfica se utilizaron diferentes bases de datos electrónicas: CSIC (IME), PubMed, Cochrane Library Plus, Dialnet y PEDro ("The Physiotherapy Data base")

Las palabras clave que se utilizaron en estas bases de datos fueron siempre las mismas: Arthroplasty, knee replacement AND CPM Therapy, Total knee replacement AND physical therapy, Gonarthrosis AND rehabilitation AND continuous passive motion, Rehabilitation AND continuous passive movement.

En la base de datos Dialnet y CSIC, la búsqueda de la información se tuvo que realizar en español y para ello se utilizaron los siguientes descriptores: Artroplastia de rodilla y CPM terapia, reemplazo total de rodilla y terapia física, gonartrosis, rehabilitación y movimiento pasivo continuo, rehabilitación y movilización pasiva continua.

\section{Selección de los estudios}

Dos revisores independientes realizaron una crítica de los artículos encontrados. En caso de desacuerdo, se empleó una puesta en común de los resultados y se llegó a un consenso entre ambos.

En primer lugar, se realizó una preselección de aquellos estudios que se mostraban potencialmente acordes con la temática.

Finalizada la búsqueda, se procedió a la lectura de los resúmenes de cada estudio, excluyéndose aquellos que no cumplieran con los criterios de inclusión anteriormente mencionados. Las publicaciones que superaron los criterios anteriores fueron sometidas a su lectura completa para el posterior análisis e inclusión definitiva en esta revisión.

Los estudios que cumplieron los criterios de inclusión y que fueron aceptados para esta revisión, se les realizó una valoración de su calidad metodológica. 
Para esta valoración se utilizó la escala PEDro $^{12}$ (Physiotherapy Evidence Data base) que indica la calidad de los ensayos. Está compuesta por 11 criterios con respuestas "SI" (S) o "NO" (N) y un rango de puntuación que varía de 0 a 10 según tengan una calidad metodológica baja, moderada o excelente ${ }^{13}$.

Los estudios se consideran de alta calidad, si la puntuación es mayor a 5 (6-8: bueno, 9-10 excelente); calidad moderada, si la puntuación es de 4 o 5 (estudio justo) y de baja calidad, si la puntuación es inferior a 4 (estudio pobre).

Los 11 criterios que se evalúan con la escala PEDro son: Especificación de los criterios de elegibilidad; Asignación aleatoria; Asignación ocultada; Base comparable; Paciente "enmascarado"; Terapeuta "enmascarado" Evaluador "enmascarado"; Seguimiento del sujeto (al menos $85 \%$ de seguimiento); Análisis del tipo intención de tratar; Comparaciones estadísticas entre los grupos y Medida de variabilidad y punto de medidas.

\section{RESULTADOS}

De los 537 estudios encontrados, fueron seleccionados 12 (Tabla 1).

Tabla 1. Descripción de los estudios incluidos

\begin{tabular}{|c|c|c|c|c|c|}
\hline Autor/a & Objetivo del estudio & $\begin{array}{l}\text { Tipo de intervención } \\
\text { muestra de estudio }\end{array}$ & $\begin{array}{l}\text { Duración de la } \\
\text { intervención y medidas }\end{array}$ & $\begin{array}{l}\text { Instrumentos de } \\
\text { valoración }\end{array}$ & Resultados \\
\hline $\begin{array}{l}\text { Leach Wy } \\
\text { coll }^{19} .2006\end{array}$ & $\begin{array}{l}\text { Investigar la influencia } \\
\text { del uso de CPM en la } \\
\text { flexión y extensión de } \\
\text { rodilla, niveles de dolor } \\
\text { y el uso de analgésicos } \\
\text { después de la cirugía } \\
\text { de PTR. }\end{array}$ & $\begin{array}{l}\text { - GC (44): ejercicios en tablero } \\
\text { deslizante y ejercicios de forta- } \\
\text { lecimiento de cuádriceps. } \\
\text { Comenzaron el día } 1 \text { posto- } \\
\text { peratorio; al día } 3 \text { se animó a } \\
\text { conseguir los } 90^{0} \text { de flexión, y } \\
\text { el alta se preveía a los } 5-7 \text { días. } \\
\text { - GE (38): siguió el mismo } \\
\text { protocolo anterior, pero se les } \\
\text { añadió CPM (1h/2veces al día). } \\
\text { Cada día se incrementaba en } \\
10^{\circ} \text { el rango de movimiento. }\end{array}$ & $\begin{array}{l}\text { Medidas: } \\
\text { - ROM en flex } \\
\text { - ROM en ext } \\
\text { - Dolor } \\
\text { - Uso de analgésicos } \\
\text { Evaluación: } \\
\text { - Preoperatoria } \\
\text { - Al alta hospitalaria } \\
\text { - } 6 \text { semanas después de } \\
\text { la operación } \\
\text { - A los } 6 \text { meses } \\
\text { - Al año de la operación }\end{array}$ & $\begin{array}{l}\text { - Goniómetro } \\
\text { - EVA } \\
\text { - Escala de la OMS }\end{array}$ & $\begin{array}{l}\text { No hubo diferencia significativa entre } \\
\text { los grupos en ROM en flex y ext. El GE } \\
\text { no experimentó una mayor tendencia } \\
\text { a la pérdida de ext en el postoperato- } \\
\text { rio temprano. } \\
\text { No hubo diferencia significativa entre } \\
\text { los grupos en el dolor. GE y GC repor- } \\
\text { taron un nivel de dolor por debajo de } \\
1 \text { en un año postoperatorio. }\end{array}$ \\
\hline $\begin{array}{l}\text { Bruun-Olsen } \\
\mathrm{y} \mathrm{col}^{20} .2009\end{array}$ & $\begin{array}{l}\text { Investigar si la CPM tie- } \\
\text { ne algún efecto sobre el } \\
\text { dolor, ROM de rodilla y } \\
\text { la capacidad de caminar, } \\
\text { en pacientes con ATR y } \\
\text { si existe relación entre el } \\
\text { ROM de rodilla y la capa- } \\
\text { cidad de caminar. }\end{array}$ & $\begin{array}{l}\text { - GC (35): ejercicios activos } \\
\text { basados en flexo-extensión } \\
\text { activa asistida de rodilla y ca- } \\
\text { dera, contracción isométrica } \\
\text { de cuádriceps, bipedestación, } \\
\text { marcha con andador o mule- } \\
\text { tas y subir/bajar escaleras con } \\
\text { muletas.30min/día. } \\
\text { - GE (35): Se les administró el } \\
\text { mismo protocolo de ejercicios } \\
\text { junto con un tratamiento de } \\
\text { CPM para la flexión y la exten- } \\
\text { sión de rodilla de manera cons- } \\
\text { tante por } 2 \mathrm{~h} / \text { día. }\end{array}$ & $\begin{array}{l}\text { Medidas: } \\
\text { - Dolor } \\
\text { - Perímetro de la rodilla } \\
\text { - ROM en flex activa y } \\
\text { pasiva } \\
\text { - ROM en ext } \\
\text { - Bipedestación y } \\
\text { caminar } \\
\text { Evaluación: } \\
\text { - Preoperatoria } \\
\text { - Una semana de la } \\
\text { operación } \\
\text { - Tres meses de la } \\
\text { operación. }\end{array}$ & $\begin{array}{l}\text { - EVA } \\
\text { - Goniómetro } \\
\text { - Cinta métrica } \\
\text { - TUG }\end{array}$ & $\begin{array}{l}\text { No hubo diferencias significativas } \\
\text { entre los dos grupos en cuanto a do- } \\
\text { lor, ROM e hinchazón a la semana y a } \\
\text { los tres meses de la intervención en } \\
\text { comparación con los datos preope- } \\
\text { ratorios. } \\
\text { Tampoco hubo diferencias en la capa- } \\
\text { cidad de caminar. }\end{array}$ \\
\hline $\begin{array}{l}\text { Maniar RN y } \\
\mathrm{col}^{21} \cdot 2012\end{array}$ & $\begin{array}{l}\text { Evaluar el efecto del uso } \\
\text { de CPM tras ATR me- } \\
\text { diante la evaluación de } \\
\text { parámetros específicos. }\end{array}$ & $\begin{array}{l}\text { - GC(28): solo recibió terapia } \\
\text { física } \\
\text { - GE1(28): terapia física junto } \\
\text { con } 2 \text { sesiones de } 15 \text { min cada } \\
\text { una de CPM desde el } 2^{\circ} \text { día } \\
\text { postoperatorio. } \\
\text { - GE2(28): terapia física y } 2 \text { se- } \\
\text { siones de } 15 \text { min cada una de } \\
\text { CPM durante tres días. }\end{array}$ & $\begin{array}{l}\text { Medidas: } \\
\text { - Dolor } \\
\text { - ROM activa } \\
\text { - Bipedestación y } \\
\text { caminar } \\
\text { - Perímetro de la rodilla } \\
\text { - Cicatrización } \\
\text { Evaluación: } \\
\text { - Preoperatoria } \\
\text { - A los } 3,5,14,42 \text { y } 90 \\
\text { días de la cirugía (día de } \\
\text { la cirugía: } 0 \text { ) }\end{array}$ & $\begin{array}{l}\text { - EVA } \\
\text { - Goniómetro } \\
\text { - TUG } \\
\text { - Cinta métrica } \\
\text { - WOMAC } \\
\text { - SF-12 }\end{array}$ & $\begin{array}{l}\text { Dolor: No diferencias estadísticamen- } \\
\text { te significativas entre los tres grupos. } \\
\text { ROM activa de la rodilla: A los } 42 \\
\text { días de la operación, el ROM en cada } \\
\text { grupo volvió al valor preoperatorio. } \\
\text { Test Up and Go: A los } 90 \text { días de la } \\
\text { operación los resultados de los tres } \\
\text { grupos fueron estadísticamente sig- } \\
\text { nificativos en comparación con los } \\
\text { datos preoperatorios. } \\
\text { Perímetro de la rodilla: GE } 1 \text { y } 2 \text { tar- } \\
\text { dó hasta } 3 \text { meses en volver a los datos } \\
\text { preoperatorios, mientras que en el GC } \\
\text { se produjo a los } 42 \text { días. } \\
\text { Cicatrización de heridas: Se observó } \\
\text { una mayor tendencia a la tinción de } \\
\text { las heridas en los GE } 1 \text { y } 2 \text {. En ninguno } \\
\text { de los tres grupos hubo complicacio- } \\
\text { nes de la herida. }\end{array}$ \\
\hline
\end{tabular}




\begin{tabular}{|c|c|c|c|c|c|}
\hline Autor/a & Objetivo del estudio & $\begin{array}{c}\text { Tipo de intervención } \\
\text { muestra de estudio }\end{array}$ & $\begin{array}{c}\text { Duración de la } \\
\text { intervención y medidas }\end{array}$ & $\begin{array}{l}\text { Instrumentos de } \\
\text { valoración }\end{array}$ & Resultados \\
\hline $\begin{array}{l}\text { Bennett LA y } \\
\mathrm{col}^{23} .2005\end{array}$ & $\begin{array}{l}\text { Evaluar el efecto de la } \\
\text { CPM después de la ATR } \\
\text { y ver si su uso al princi- } \\
\text { pio de la flexión produce } \\
\text { mejor resultado que un } \\
\text { tratamiento sin CPM. }\end{array}$ & $\begin{array}{l}\text { - GC (52): protocolo fisiotera- } \\
\text { pia (ejercicios activos miem- } \\
\text { bro inferior y contracciones } \\
\text { isométricas). } 30 \text { min 2veces/ } \\
\text { día excepto fines de semana } \\
\text { - GE } 1 \text { (47): protocolo fisiotera- } \\
\text { pia } 30 \text { min } 2 \text { veces/día excepto } \\
\text { fines de semana más CPM } 3 \mathrm{~h} 2 \\
\text { veces/día y férula en extensión } \\
\text { nocturna. } \\
\text { - GE2 (48): protocolo de fisiote- } \\
\text { rapia } 30 \text { min } 2 \text { veces/día excep- } \\
\text { to fines de semana y CPM con } \\
\text { la misma pauta pero sin férula } \\
\text { en extensión hasta el } 5^{\circ}-6^{0} \text { día. }\end{array}$ & $\begin{array}{l}\text { Medidas: } \\
\text { - ROM de flex } \\
\text { - ROM de ext } \\
\text { - Dolor } \\
\text { - Funciónalidad rodilla } \\
\text { - Estado de salud } \\
\text { percibido por los } \\
\text { pacientes } \\
\text { - Duración de la estancia } \\
\text { - Cicatrización } \\
\text { Evaluación: } \\
\text { - Preoperatorio } \\
\text { - Postoperatorio } \\
\text { - A los 5 días } \\
\text { - A los tres meses } \\
\text { - Y al año de la misma. } \\
\end{array}$ & $\begin{array}{l}\text { - Goniómetro } \\
\text { - SF-12 } \\
\text { - KSS } \\
\text { - EVA }\end{array}$ & $\begin{array}{l}\text { ROM: No hallaron diferencias signifi- } \\
\text { cativas entre los tres grupos en ningu- } \\
\text { na de las evaluaciones, en cuanto a la } \\
\text { flexi activa, ext pasiva y la fuerza de } \\
\text { cuádriceps. } \\
\text { Dolor: Hubo diferencias significativas } \\
\text { entre GE } 1 y 2 ; 2 \text { y GC; y } 1 \text { y GC; pero } \\
\text { no eran clínicamente significativas } \\
\text { porque presentan un valor } \leq 1 \text { en una } \\
\text { escala de } 10 \\
\text { Duración de la estancia, Cicatriza- } \\
\text { ción de las heridas y función de la } \\
\text { rodilla: No hubo diferencias significa- } \\
\text { tivas entre los grupos. }\end{array}$ \\
\hline $\begin{array}{l}\text { Denis M y } \\
\mathrm{col}^{22} \cdot 2006\end{array}$ & $\begin{array}{l}\text { Comparar la eficacia de } \\
\text { tres programas de reha- } \\
\text { bilitación con diferentes } \\
\text { intensidades de aplica- } \\
\text { ción de CPM tras ATR. }\end{array}$ & $\begin{array}{l}\text { - GC(27): protocolo fisioterapia } \\
\text { (ejercicios respiratorios, iso- } \\
\text { métricos, de carga, escaleras) } \\
\text { - GE } 1 \text { (26): mismo protocolo } \\
\text { de fisioterapia y } 35 \text { min diarios } \\
\text { de CPM de intensidad baja. } \\
\text { - GE } 2 \text { (28): mismo protocolo } \\
\text { de fisioterapia y } 2 \text { h diarias de } \\
\text { CPM con intensidad moderada. }\end{array}$ & $\begin{array}{l}\text { Medidas: } \\
\text { - ROM activa de flexión } \\
\text { - ROM de extensión } \\
\text { - Bipedestación, caminar, } \\
\quad \text { girar y sentarse } \\
\text { - Dolor } \\
\text { - Duración de la estancia } \\
\text { Evaluación: } \\
\text { - Preoperatoria } \\
\text { - Y al alta } \\
\end{array}$ & $\begin{array}{l}\text { - Goniómetro } \\
\text { - TUG } \\
\text { - WOMAC } \\
\text { - EVA }\end{array}$ & $\begin{array}{l}\text { No obtuvieron diferencias estadística- } \\
\text { mente significativas entre los tres gru- } \\
\text { pos ni al inicio ni al alta en ninguna de } \\
\text { las medidas que realizaron. }\end{array}$ \\
\hline $\begin{array}{l}\text { Lenssen TA y } \\
\operatorname{col}^{16} .2008\end{array}$ & $\begin{array}{l}\text { Establecer si existe be- } \\
\text { neficio adicional con la } \\
\text { aplicación de CPM tras } \\
\text { el alta hospitalaria a lar- } \\
\text { go plazo. }\end{array}$ & $\begin{array}{l}\text { - GC (30): protocolo fisiotera- } \\
\text { pia (movilización pasiva y acti- } \\
\text { va de la rodilla, fortalecimiento } \\
\text { activo de cuádriceps y forma- } \\
\text { ción en AVD) tanto en su estan- } \\
\text { cia en el hospital como las dos } \\
\text { primeras semanas después del } \\
\text { alta (30min/sesión) } \\
\text { - GE (30): protocolo fisiotera- } \\
\text { pia y CPM durante los } 17 \text { días } \\
\text { siguientes a la cirugía. } \\
\text { A partir del día } 18 \text { hasta los } 3 \\
\text { meses, ambos grupos recibie- } \\
\text { ron fisioterapia. }\end{array}$ & $\begin{array}{l}\text { Medidas: } \\
\text { - Funcionalidad rodilla } \\
\text { - Uso de medicación } \\
\text { - ROM total } \\
\text { - Satisfacción con } \\
\text { el tratamiento y el } \\
\text { resultado } \\
\text { - Adhesión de los } \\
\text { protocolos de } \\
\text { tratamiento y uso CPM } \\
\text { - Cantidad, duración } \\
\text { y naturaleza de la } \\
\text { intervención de } \\
\text { fisioterapia. } \\
\text { Evaluación: } \\
\text { - Preoperatoria } \\
\text { - Al alta } \\
\text { - A los } 17 \text { días } \\
\text { - Seis semanas } \\
\text { - } 3 \text { meses de la cirugía. } \\
\end{array}$ & $\begin{array}{l}\text { - WOMAC } \\
\text { - KSS } \\
\text { - Escala de Likert } \\
\text { - Goniómetro } \\
\text { - Diario del } \\
\quad \text { paciente }\end{array}$ & $\begin{array}{l}\text { ROM total: Mayor tendencia a favor } \\
\text { del uso prolongado de CPM al final del } \\
\text { período de tratamiento, ya que el GE } \\
\text { logró } 5^{\circ}-6^{\circ} \text { más de ROM que el grupo } \\
\text { control. A las } 6 \text { semanas y a los } 3 \text { me- } \\
\text { ses no se encontraron diferencias en } \\
\text { el ROM. } \\
\text { Estado funcional: En un principio el } \\
\text { GE obtuvo mejor puntuación en el KSS } \\
\text { y en el WOMAC. No hubo diferencias } \\
\text { significativas entre los dos grupos ni } \\
\text { en el día } 17 \text { ni en ninguna de las si- } \\
\text { guientes evaluaciones. } \\
\text {-Respecto al resto de las medidas no } \\
\text { se encontraron diferencias significa- } \\
\text { tivas. }\end{array}$ \\
\hline $\begin{array}{l}\text { Chen LH y } \\
\text { coll }^{14} .2013\end{array}$ & $\begin{array}{l}\text { Evaluar los efectos de } \\
\text { CMP en el rango de movi- } \\
\text { miento, dolor postopera- } \\
\text { torio y calidad de vida de } \\
\text { los pacientes sometidos } \\
\text { a artroplastia total de ro- } \\
\text { dilla en los } 6 \text { meses pos- } \\
\text { teriores a la operación. } \\
\end{array}$ & $\begin{array}{l}\text { - GC: Protocolo básico de reha- } \\
\text { bilitación tras artroplastia. } \\
\text { - GE: Protocolo básico de reha- } \\
\text { bilitación tras artroplastia más } \\
\text { 6horas/día de CPM. }\end{array}$ & $\begin{array}{l}\text { Medidas: } \\
\text { - ROM } \\
\text { - Dolor } \\
\text { Evaluación: } \\
\text { - Preoperatorio } \\
\text { - A los } 6 \text { meses de la } \\
\quad \text { operación. }\end{array}$ & $\begin{array}{l}\text { - Goniómetro } \\
\text { - EVA } \\
\text { - SF-36 }\end{array}$ & $\begin{array}{l}\text { CPM no proporciona beneficios ob- } \\
\text { vios tras la artroplastia total de ro- } \\
\text { dilla. }\end{array}$ \\
\hline $\begin{array}{l}\text { Mau-Moeller } \\
\text { A y coll } \\
2014\end{array}$ & $\begin{array}{l}\text { Comparar los efectos } \\
\text { producidos por CPM en } \\
\text { comparación con fisio- } \\
\text { terapia convencional } \\
\text { tras ATR. }\end{array}$ & $\begin{array}{l}\text { - GC (19): recibió fisioterapia } \\
\text { convencional más CPM. } 30 \text { mi- } \\
\text { nutos/día desde postoperato- } \\
\text { rio durante } 3 \text { semanas. } \\
\text { - GE (19): Recibió fisioterapia } \\
\text { convencional más ejercicios de } \\
\text { entrenamiento. } 30 \text { minutos/día } \\
\text { desde postoperatorio durante } \\
3 \text { semanas. }\end{array}$ & $\begin{array}{l}\text { Medidas: } \\
\text { - ROM pasiva de flex y ext } \\
\text { - ROM activa de flex y ext } \\
\text { - Control postural } \\
\text { estático } \\
\text { - Actividad física, dolor } \\
\text { - Estancia hospitalaria. } \\
\text { - Estado funcional } \\
\text { - Calidad de vida } \\
\text { Evaluación: } \\
\text { - Preoperatorio } \\
\text { - Al día siguiente } \\
\text { operación } \\
\text { - A los 3 meses de la } \\
\text { operación. }\end{array}$ & $\begin{array}{l}\text { - Goniómetro } \\
\text { - EVA } \\
\text { - Monitor de } \\
\quad \text { actividad } \\
\text { - Placa estática de } \\
\quad \text { control postural } \\
\text { - SF-36 } \\
\text { - KSS } \\
\text { - WOMAC }\end{array}$ & $\begin{array}{l}\text { Ejercicios de entrenamiento parecen } \\
\text { tener un efecto a corto plazo más be- } \\
\text { neficioso en comparación con CPM. } \\
\text { Los resultados apoyan la implemen- } \\
\text { tación del ejercicio de entrenamiento } \\
\text { en los programas de rehabilitación } \\
\text { después de la ATR. }\end{array}$ \\
\hline
\end{tabular}




\begin{tabular}{|c|c|c|c|c|c|}
\hline Autor/a & Objetivo del estudio & $\begin{array}{l}\text { Tipo de intervención } \\
\text { muestra de estudio }\end{array}$ & $\begin{array}{c}\text { Duración de la } \\
\text { intervención y medidas }\end{array}$ & $\begin{array}{l}\text { Instrumentos de } \\
\text { valoración }\end{array}$ & Resultados \\
\hline $\begin{array}{l}\text { Herbold JA y } \\
\mathrm{col}^{17} \cdot 2014\end{array}$ & $\begin{array}{l}\text { Determinar los efectos } \\
\text { del uso de un movi- } \\
\text { miento pasivo continuo } \\
\text { (CPM) en el rango de } \\
\text { movimiento (ROM) tras } \\
\text { PTR. }\end{array}$ & $\begin{array}{l}\text { - GC (71): recibió } 3 \text { horas/día } \\
\text { de terapia convencional. } \\
\text { - GE (70): } 3 \text { horas/día de tera- } \\
\text { pia convencional más 2horas/ } \\
\text { día de CPM }\end{array}$ & $\begin{array}{l}\text { Medidas: } \\
\text { - ROM activa de flex } \\
\text { - ROM de ext } \\
\text { - Estado funcional } \\
\text { - Perímetro de la rodilla } \\
\text { - Índice de osteoartritis } \\
\text { - Estancia hospitalaria } \\
\text { Evaluación: } \\
\text { - Preoperatorio } \\
\text { - A los } 8 \text { días } \\
\text { - Al alta }\end{array}$ & $\begin{array}{l}\text { - WOMAC } \\
\text { - TUG } \\
\text { - Goniómetro } \\
\text { - FIM } \\
\text { - Cinta métrica }\end{array}$ & $\begin{array}{l}\text { CPM no proporciona un beneficio adi- } \\
\text { cional tras las intervenciones conven- } \\
\text { cionales en pacientes con mala ROM } \\
\text { de flex. de la rodilla después de la } \\
\text { cirugía inicial. } \\
\text { ROM total: Los pacientes de ambos } \\
\text { grupos mostraron una mejora signifi- } \\
\text { cativa en la flexo-extensión de rodilla. } \\
\text { Se produjo una mejora de la percep- } \\
\text { ción del dolor y (WOMAC) tras el } \\
\text { tratamiento. }\end{array}$ \\
\hline $\begin{array}{l}\text { Boese CK y } \\
\mathrm{col}^{25} .2014\end{array}$ & $\begin{array}{l}\text { Evaluar los efectos de } \\
\text { uso a corto plazo de } \\
\text { CPM en comparación } \\
\text { con fisioterapia están- } \\
\text { dar tras PTR. }\end{array}$ & $\begin{array}{l}\text { - GC (29): CPM inmediatamen- } \\
\text { te después de la operación. } 5 \\
\text { horas diarias, mínimo } 2 \text { días. } \\
\text { Hasta alcanzar } 90^{\circ} \text { de flex. ac- } \\
\text { tiva. } \\
\text { - GE1 1(25): Pierna fija en } 90^{\circ} \\
\text { flexión rodilla durante } 8-19 \\
\text { horas tras operación. Después } \\
\text { CPM 5h/día hasta alta hospi- } \\
\text { talaria. } \\
\text { - GE2(22): Reposo durante la } \\
\text { estancia hospitalaria. }\end{array}$ & $\begin{array}{l}\text { Medidas: } \\
\text { - ROM activa de flex } \\
\text { - ROM de ext } \\
\text { - Dolor } \\
\text { - Nivel de hemoglobina } \\
\text { - Perímetro de la rodilla } \\
\text { - Estancia hospitalaria } \\
\text { Evaluación: } \\
\text { - Preoperatorio } \\
\text { - Cada día de } \\
\text { postoperatorio durante } \\
\text { la estancia hospitalaria. } \\
\text { - A las } 3 \text { semanas del alta } \\
\text { hospitalaria. }\end{array}$ & $\begin{array}{l}\text { - Goniómetro } \\
\text { - Cinta métrica } \\
\text { - Análisis } \\
\text { sanguíneo } \\
\text { hemoglobina. }\end{array}$ & $\begin{array}{l}\text { CPM no proporciona beneficio tras } \\
\text { la intervención de PTR, no mejora la } \\
\text { movilidad precoz ni reduce la estan- } \\
\text { cia hospitalaria. } \\
\text { ROM total y perímetro de la rodilla: } \\
\text { NO se observaron diferencias signif- } \\
\text { cativas entre los grupos. } \\
\text {-GE } 2 \text { fue el que tuvo un mayor aumen- } \\
\text { to del perímetro, manifestándose al } 2^{9} \\
\text { día de la operación. }\end{array}$ \\
\hline $\begin{array}{l}\text { Alkire MR y } \\
\mathrm{col}^{24} .2010\end{array}$ & $\begin{array}{l}\text { Determinar si CPM } \\
\text { asistida por ordena- } \\
\text { dor tras PTR produjo } \\
\text { diferencias en amplitud } \\
\text { de movimiento, edema, } \\
\text { capacidad funcional } \\
\text { y dolor }\end{array}$ & $\begin{array}{l}\text { - GC: Terapia física } 2 \text { veces/ } \\
\text { día más } \\
\text { - GE: Terapia física } 2 \text { veces/día } \\
\text { más terapia CPM } 3 \text { veces/día. }\end{array}$ & $\begin{array}{l}\text { Medidas: } \\
\text { - Funcionalidad rodilla } \\
\text { - Îndice de osteoartritis } \\
\text { - ROM } \\
\text { - Perímetro rodilla } \\
\text { - Edema } \\
\text { Evaluación: } \\
\text { - Preoperatrorio } \\
\text { - A los } 3 \text { meses }\end{array}$ & $\begin{array}{l}\text { - KSS } \\
\text { - WOMAC } \\
\text { - Goniómetro } \\
\text { - Cinta métrica } \\
\text { - Análisis } \\
\text { sanguíneo } \\
\text { hemoglobina }\end{array}$ & $\begin{array}{l}\text { No hubo diferencia estadísticamente } \\
\text { significativa en la flex, edema o dre- } \\
\text { naje, función y dolor entre los grupos } \\
\text { durante el período de estudio de } 3 \\
\text { meses. }\end{array}$ \\
\hline $\begin{array}{l}\text { Davies DM, y } \\
\text { col }^{18} .2003\end{array}$ & $\begin{array}{l}\text { Determinar si el uso } \\
\text { de CPM junto con el } \\
\text { tratamiento estándar } \\
\text { contribuye a reducir la } \\
\text { hospitalización y mejora } \\
\text { de pacientes sometidos } \\
\text { a ATR. }\end{array}$ & $\begin{array}{l}\text { - GC (40): CPM } 2 \text { horas al día } \\
\text { junto con programa de ejerci- } \\
\text { cios convencionales de } 30 \text { mi- } \\
\text { nutos diarios. } \\
\text { - GE1 (40): Ejercicios con plata- } \\
\text { forma de deslizamiento duran- } \\
\text { te } 10 \text { minutos diarios junto con } \\
\text { programa de ejercicios conven- } \\
\text { cionales de } 30 \text { minutos diarios. } \\
\text { - GE } 2 \text { ( } 40) \text { : Programa de ejer- } \\
\text { cicios convencionales de } 30 \\
\text { minutos diarios. } \\
\text { Ejercicios convencionales } \\
\text { comienzan al } 3^{o} \text { día de la ope- } \\
\text { ración. } \\
\text { CPM comienza al } 2^{0} \text { día de la } \\
\text { operación. } \\
\text { Ejercicios con tabla de desliza- } \\
\text { miento comienza al } 3^{\circ} \text { día y se } \\
\text { utiliza simultáneamente duran- } \\
\text { te la realización del programa } \\
\text { de ejercicios. }\end{array}$ & $\begin{array}{l}\text { Medidas: } \\
\text { - ROM } \\
\text { - Estancia hospitalaria } \\
\text { - Independencia en la } \\
\text { marcha y trasferencias } \\
\text { Evaluación: } \\
\text { - Preoperatorio } \\
\text { - A los } 3 \text { meses. } \\
\text { - A los } 6 \text { meses. }\end{array}$ & $\begin{array}{l}\text { - Goniómetro } \\
\text { - Visitas al hospital }\end{array}$ & $\begin{array}{l}\text { Después de la operación, los } 3 \text { grupos } \\
\text { de tratamiento mostraron un patrón } \\
\text { de recuperación muy similar. }\end{array}$ \\
\hline
\end{tabular}

GE: grupo experimental; GC: grupo control; Flex.: flexión; Ext: extensión; CPM: Movilización Pasiva Continua; PTR: Prótesis Total de Rodilla; ROM: Range Of Movement; EVA: Escala Visual Analógica; OMS: Organización Mundial de la Salud; ATR: Artroplastia Total de Rodilla; WOMAC: Western Ontario and McMaster Universities Arthritis Index; FIM: Medida de Independencia Funcional; HSS: Hospital for Special Surgery; KSS: Knee Society Clinical Rating System; TUG: Test Up and Go. 
El proceso de búsqueda y la selección de los estudios que se incluyeron y excluyeron en esta revisión sistemática quedan reflejados en la figura 1 .

\section{Descripción de los artículos}

Los estudios incluidos contenían información de un total de 1.153 pacientes que iban a ser sometidos a una artroplastia total de rodilla por osteoartrosis u osteoartritis, donde se identificaron sus características, el tiempo de permanencia en el hospital, momento de inicio y duración del tratamiento postquirúrgico, tiempo de aplicación de las técnicas empleadas, la administración de analgésicos, y las medidas de resultado y los efectos obtenidos.
En estos estudios, la muestra de sujetos que se utiliza es variable. Siendo la mayor muestra, $\mathrm{N}=170$, del trabajo realizado por Chen y coll $^{14}$ y la menor muestra, $\mathrm{N}=38$, la utilizada en la investigación de Mau-Moeller A. y col ${ }^{15}$.

En todos los trabajos analizados se tomaron medidas en cuanto al rango de movilidad articular, tanto en flexión como extensión ${ }^{14-25}$. El dolor fue el segundo parámetro más estudiado, siendo valorado en todos los trabajos excepto en los realizados por Lenssen $\mathrm{y} \mathrm{col}^{16}$, Herbold y $\operatorname{col}^{17}$ y Davies y col ${ }^{18}$. En cada estudio se añadieron además otras medidas secundarias como el uso de analgésicos ${ }^{19}$, hinchazón ${ }^{20,21}$, la bipedestación y caminar ${ }^{20-22}$, cicatrización de las heridas ${ }^{21,23}$, función de

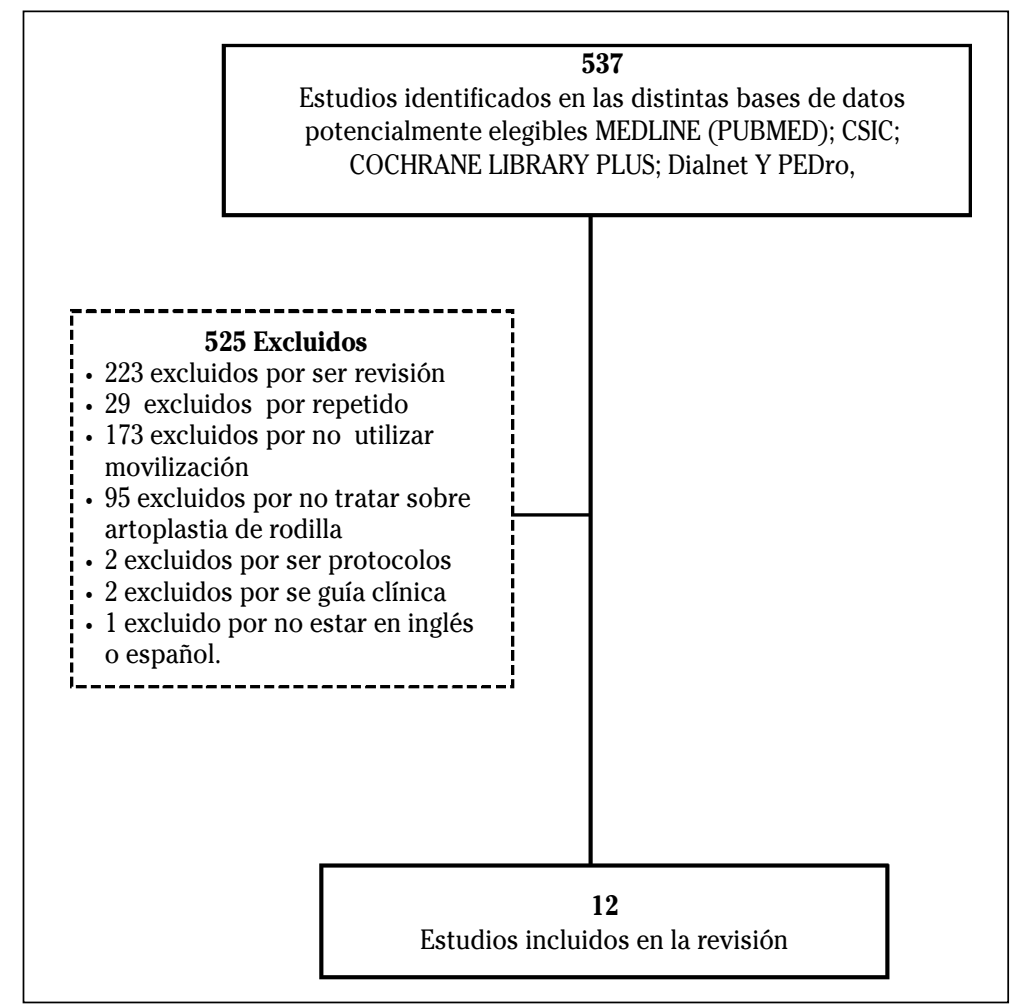

Figura 1. Procedimiento de selección de los artículos en las búsquedas bibliográficas. 
la rodilla ${ }^{15-17,23,24}$,estado de salud percibido por los pacientes ${ }^{23}$, duración de la estancia hospitalaria ${ }^{15,17,18,22,23,26}$, estado funcional ${ }^{16}$, uso de medicación ${ }^{16}$, satisfacción con el tratamiento y su resultado ${ }^{16}$, adhesión de los protocolos de tratamiento y uso de la movilización pasiva continua ${ }^{16}$, y la cantidad, duración y naturaleza de la intervención de fisioterapia ${ }^{16}$.

En todos los estudios ${ }^{14-25}$ se realizó la medida de los parámetros analizados antes de que los pacientes fueran sometidos a la artroplastia, es en la temporalización del resto de valoraciones donde más difieren unos de otros. Los trabajos realizados por Leach y col $^{19}$ y por Denis y col $^{22}$ coinciden en realizar una nueva valoración de los parámetro de estudio en el momento del alta hospitalaria. En el resto de trabajos estas mediciones de seguimiento se realizan a las 6 semanas $^{16,19,21}$, a los 3 meses $^{15,16,18,20,21,24}$,y a los 6 meses de la intervención ${ }^{14,18,19}$. Las mediciones de seguimiento más exhaustivas fueron las realizadas por Maniar RN y $\mathrm{col}^{21}$ ya que valoraron al paciente a los a los 3, 5, 14, 42 y 90 días de la intervención, siendo las más prolongadas en el tiempo las realizadas por Leach y $\operatorname{col}^{19}$ y Bennett $\mathrm{y}$ $\mathrm{col}^{23}$ que realizaron la última valoración de seguimiento al año de la cirugía.

En todos los estudios hay un grupo control al que se le aplica el tratamiento de fisioterapia y un grupo experimental al que, además del tratamiento de fisioterapia, se añade el tratamiento con movilización pasiva continua. No obstante, en algunas investigaciones ${ }^{18,21-23}$ se añade un segundo grupo experimental al que también se le aplica el tratamiento de fisioterapia y movilización pasiva continua, pero con una variación en la duración de la aplicación de la misma para poder comparar los resultados entre las diferentes intervenciones. En el trabajo realizado por Boese $\mathrm{y} \mathrm{col}^{26}$ este tercer grupo control solo permanece en reposo durante su estancia hospitalaria.

Se utilizó el goniómetro para la valoración del rango de movilidad articular. Además, en cada estudio, se emplearon otros instrumentos de valoración, La escala visual analógica ${ }^{14,15,19-23}$ (EVA); la cirtome- tría ${ }^{17,20,21,24,25}$; el Test Up and Go ${ }^{17,20-22}$ (TUG); el Western Ontario McMaster Universities Osteoarthritis Index ${ }^{15-17,21,22,24}$ (WOMAC); el Short-Form 12-item Health Survey ${ }^{21,23}$ (SF12); el Short-Form 36-item Health Survey ${ }^{14,15}$ (SF-36); el componente funcional de la Knee Society Clinical Rating System o Knee Society Score ${ }^{15,16,23,24}$ (KSS); las escalas de Likert $^{16}$; la medida de la independencia funcional ${ }^{17}$ y el análisis de la hemoglobina en sangre ${ }^{24,25}$.

\section{MEDIDAS DE RESULTADOS}

\section{Amplitud de movimiento}

En ninguno de los estudios analizados existe una mejoría significativa en la flexión y extensión de la rodilla ${ }^{14-25}$ sin embargo, se ha observado que en el grupo experimental, al cual se le aplicaba movilización pasiva continua, no se experimentaba una mayor tendencia a la pérdida de extensión en el postoperatorio inmediato ${ }^{19}$.

Se han visto ligeros efectos positivos en el incremento del rango articular en aquellos pacientes que han recibido el tratamiento de fisioterapia junto con aplicaciones de movilización pasiva continua, consiguiendo $5^{\circ}-6^{\circ}$ más de amplitud articular durante el periodo de tratamiento. Sin embargo, tanto a las 6 semanas como a los 3 meses tras la intervención no se ha detectado ninguna diferencia significativa entre las dos estrategias de tratamiento. Por lo tanto, los investigadores afirman que el uso de la movilización pasiva continua tiene un efecto a corto plazo en movilidad articu$\operatorname{lar}^{15,16}$.

Por otro lado Bennett y $\mathrm{col}^{23}$ y MauMoeller $\mathrm{y} \mathrm{col}^{15}$ son los únicos autores que, según su estudio, establecieron que la aplicación de un régimen de movilización pasiva continua en el postoperatorio temprano puede resultar útil para la consecución de un mejor rango de flexión temprana tras la artroplastia total de rodilla.

En los trabajos realizados por Chen y $\mathrm{col}^{14}$, Herbold y $\mathrm{col}^{17}$, Boese y col${ }^{25}$, Alkire ${ }^{24}$, y Davies y $\operatorname{col}^{18}$ se afirma que la CMP no proporciona beneficios adicionales en la movilidad articular. 


\section{Dolor}

No se encontraron diferencias significativas en la reducción de la intensidad entre los sujetos de los grupos de intervención con respecto al grupo control en todos los intervalos que se establecieron mediciones, ya sean inmediatas tras la operación, al tercer o quinto día ${ }^{21} \mathrm{o}$ en las realizadas tras un año del postoperatorio ${ }^{19}$.

Sin embargo, algunos trabajos encontraron que al aplicar esta técnica se producía una disminución del dolor ${ }^{20,23}$ que se mantenía hasta los tres meses después de la intervención ${ }^{20}$. En los estudios restantes $^{14-18,22,24,25}$ no se especifican los resultados obtenidos en la valoración del dolor.

\section{Perímetro de la rodilla}

Tras la intervención, no se observaron diferencias significativas en el perímetro de la rodilla entre los grupos participantes.

Maniar RN y col$^{21}$ afirman en su trabajo que la inflamación de la rodilla fue más pronunciada al tercer día $(2-3 \mathrm{~cm})$, tardando hasta 3 meses en recuperar su diámetro en los grupos experimental 1 y 2 , ambos tratados con CPM; el grupo control logró la recuperación de este parámetro a los 42 días.

Estos resultados son similares a los obtenidos por Boese $\mathrm{CK}$ y col${ }^{25}$. En dicho trabajo el grupo tratado con CPM 5h/día más mantenimiento de flexión fue el que obtuvo un mayor incremento del perímetro de la rodilla, obteniéndose éste al segundo día de la operación.

Se ha visto que en algunos casos a partir del quinto día los valores de inflamación se han reducido en todos los grupos (experimental y control), y que tras los 42 días de la intervención el grado de inflamación se ha incrementado en los pacientes del grupo control hasta nivelarse a los valores preoperatorios, sin embargo los sujetos del grupo experimental los han alcanzado a los 3 meses de la intervención. Por lo tanto, el uso de la movilización pasiva continua retrasó la recuperación de la inflamación de la rodilla.

\section{Test Up and Go}

Este test se utilizó para la valoración de la bipedestación y de la marcha.

Bruun-Olsen y col ${ }^{20}$ no obtuvieron diferencias significativas entre los grupos de estudio en los resultados de dicho test a la semana y a los 3 meses de la operación.

Los mejores datos en los resultados de la prueba son los obtenidos en el día 90 por Maniar y col$^{21}$ los cuales observaron una mejora significativa de los resultados en los tres grupos de estudio comparándolos con valores preoperatorios. Los valores de este test fueron 5 veces superiores en el tercer día, tras la intervención, que los valores obtenidos en el preoperatorio. A partir de ese momento, los valores comenzaron a descender siendo en el quinto día cuatro veces mayores del valor preoperatorio y a los 14 días descendieron al doble de dicho valor. Sin embargo esta diferencia no fue estadísticamente significativa.

En el trabajo realizado por Denis $\mathrm{M}$ y $\mathrm{col}^{22}$ no se observan diferencias significativas entre los grupos ni en el preoperatorio ni en el momento del alta.

\section{Cicatrización}

En el trabajo realizado por Maniar RN y $\mathrm{col}^{21}$ se ha visto que al tercer día de la intervención la mayoría de sujetos de los grupos experimentales presentaban tinción de las heridas en comparación con una minoría del grupo control, incrementándose las complicaciones a partir del quinto día.

Otros estudios muestran que el uso de CPM como parte del tratamiento rehabilitador no influye en la cicatrización de la herida del paciente, no existiendo diferencias significativas entre los grupos de estudio ${ }^{23}$.

\section{Estancia hospitalaria}

La duración de la estancia hospitalaria para cada grupo, experimental o control, fue muy similar, siendo una media de 8,1 8,8 días para el primero y 7,8-8 días para el segundo $^{22,23}$. Los pacientes fueron dados de alta cuando el equipo multidisciplinar los evaluó y se aseguraron que eran capaces 
Tabla 2. Evaluación de la calidad metodológica

\begin{tabular}{|c|c|c|c|c|c|c|c|c|c|c|c|c|}
\hline Artículo & 1 & 2 & 3 & 4 & 5 & 6 & 7 & 8 & 9 & 10 & 11 & Puntuación \\
\hline Leach y col $(2006)^{19}$ & $\mathrm{~S}$ & $\mathrm{~N}$ & $\mathrm{~N}$ & $\mathrm{~N}$ & $\mathrm{~N}$ & $\mathrm{~N}$ & $\mathrm{~S}$ & $\mathrm{~N}$ & $\mathrm{~N}$ & $\mathrm{~S}$ & $\mathrm{~N}$ & 2 \\
\hline Vigdis Bruun-Olsen y col $(2009)^{20}$ & $\mathrm{~S}$ & $\mathrm{~S}$ & $\mathrm{~S}$ & $\mathrm{~N}$ & $\mathrm{~S}$ & $\mathrm{~S}$ & $\mathrm{~S}$ & $\mathrm{~S}$ & $\mathrm{~N}$ & $\mathrm{~S}$ & $\mathrm{~N}$ & 7 \\
\hline Maina $\mathrm{RN}^{21}(2012)^{21}$ & $\mathrm{~S}$ & $\mathrm{~S}$ & $\mathrm{~S}$ & $\mathrm{~N}$ & $\mathrm{~S}$ & $\mathrm{~N}$ & $\mathrm{~S}$ & $\mathrm{~S}$ & $\mathrm{~S}$ & $\mathrm{~S}$ & $\mathrm{~N}$ & 7 \\
\hline Bennett LA $(2005)^{23}$ & $\mathrm{~S}$ & $\mathrm{~S}$ & $\mathrm{~N}$ & $\mathrm{~S}$ & $\mathrm{~N}$ & $\mathrm{~N}$ & $\mathrm{~S}$ & $\mathrm{~S}$ & $\mathrm{~N}$ & $\mathrm{~S}$ & $\mathrm{~S}$ & 6 \\
\hline Denis M $(2006)^{22}$ & $\mathrm{~S}$ & $\mathrm{~S}$ & $\mathrm{~S}$ & $\mathrm{~N}$ & $\mathrm{~S}$ & $\mathrm{~S}$ & $\mathrm{~S}$ & $\mathrm{~S}$ & $\mathrm{~S}$ & $\mathrm{~S}$ & $\mathrm{~N}$ & 8 \\
\hline Lenssen TA y col (2008) ${ }^{16}$ & $\mathrm{~S}$ & $\mathrm{~S}$ & $\mathrm{~S}$ & $\mathrm{~S}$ & $\mathrm{~N}$ & $\mathrm{~N}$ & $\mathrm{~S}$ & $\mathrm{~S}$ & $\mathrm{~S}$ & $\mathrm{~S}$ & $\mathrm{~S}$ & 8 \\
\hline Chen LH y col. $(2013)^{14}$ & $\mathrm{~S}$ & $\mathrm{~N}$ & $\mathrm{~N}$ & $\mathrm{~S}$ & $\mathrm{~N}$ & $\mathrm{~N}$ & $\mathrm{~N}$ & $\mathrm{~N}$ & $\mathrm{~N}$ & $\mathrm{~S}$ & $\mathrm{~S}$ & 3 \\
\hline Mau-Moeller y col $(2014)^{14}$ & $\mathrm{~S}$ & $\mathrm{~S}$ & $\mathrm{~S}$ & $\mathrm{~S}$ & $\mathrm{~N}$ & $\mathrm{~N}$ & $\mathrm{~S}$ & $\mathrm{~S}$ & $\mathrm{~S}$ & $\mathrm{~S}$ & $\mathrm{~S}$ & 8 \\
\hline Herbold JA y col (2014) ${ }^{17}$ & $\mathrm{~S}$ & $\mathrm{~S}$ & $\mathrm{~N}$ & $\mathrm{~S}$ & $\mathrm{~N}$ & $\mathrm{~N}$ & $\mathrm{~S}$ & $\mathrm{~S}$ & $\mathrm{~N}$ & $\mathrm{~S}$ & $\mathrm{~S}$ & 6 \\
\hline Boese CK y col $(2014)^{25}$ & $\mathrm{~S}$ & $\mathrm{~S}$ & $\mathrm{~S}$ & $\mathrm{~S}$ & $\mathrm{~N}$ & $\mathrm{~N}$ & $\mathrm{~N}$ & $\mathrm{~S}$ & $\mathrm{~N}$ & $\mathrm{~S}$ & $\mathrm{~S}$ & 6 \\
\hline Alkire MR Swank ML $(2010)^{24}$ & $\mathrm{~S}$ & $\mathrm{~S}$ & $\mathrm{~N}$ & $\mathrm{~S}$ & $\mathrm{~N}$ & $\mathrm{~N}$ & $\mathrm{~N}$ & $\mathrm{~S}$ & $\mathrm{~N}$ & $\mathrm{~N}$ & $\mathrm{~N}$ & 3 \\
\hline Davies DM y col $(2003)^{18}$ & $\mathrm{~N}$ & $\mathrm{~S}$ & $\mathrm{~S}$ & $\mathrm{~S}$ & $\mathrm{~N}$ & $\mathrm{~N}$ & $\mathrm{~S}$ & $\mathrm{~S}$ & $\mathrm{~S}$ & $\mathrm{~S}$ & $\mathrm{~S}$ & 8 \\
\hline
\end{tabular}

S: presenta el criterio estudiado; $\mathrm{N}$ : no presenta el criterio estudiado.

1. Especificación de los criterios de elegibilidad; 2. Asignación aleatoria; 3. Asignación ocultada; 4. Base comparable: los grupos fueron similares al inicio con respecto a los indicadores pronósticos más importantes; 5 . Paciente "enmascarado"; 6. Terapeuta "enmascarado"; 7. Evaluador "enmascarado"; 8. Seguimiento del sujeto (al menos 85\% de seguimiento); 9. Análisis del tipo intención de tratar; 10. Comparaciones estadísticas entre los grupos; 11. Medida de variabilidad y punto de medidas

de manejarse de forma correcta en su entorno familiar ${ }^{23}$. Estos datos coinciden con los obtenidos por Herbold y $\mathrm{col}^{17}$, ya que en su estudio los pacientes de ambos grupos permanecieron una media de 8 días en el hospital.

El estudio realizado por Boese $\mathrm{y} \mathrm{col}^{25}$ es el que obtuvo una más rápida recuperación de sus pacientes, ya que la estancia media hospitalaria de los mismos fue de 2.7, $3.2 \mathrm{y}$ 2.6 días en los grupos control, y 1 y 2 en el experimental.

\section{Adherencia de las intervenciones}

Denis y col$^{22}$ decidieron evaluar la adhesión al tratamiento tanto de la movilización pasiva continua como de las intervenciones convencionales de fisioterapia, siendo en ambos casos muy alta esta adhesión. En el caso de la movilización pasiva continua sólo 4 sujetos del grupo experimental no recibieron el $75 \%$ de las intervenciones que estuvieron planificadas. Por otro lado, en el grupo de terapia física solo 3 sujetos del grupo control y un sujeto del grupo experi- mental no recibieron el 75\% de las intervenciones de fisioterapia.

\section{Estado funcional y percepción del estado de salud percibido}

Los pacientes estaban satisfechos tanto con su tratamiento individual como con los resultados del tratamiento ${ }^{16}$.

En general no se obtuvieron diferencias significativas entre el grupo experimental y control, ni tras la intervención ni en las mediciones de seguimiento que se realizaron (3-12 meses) $)^{15,16,23,24}$.

\section{Evaluación de la calidad metodológica}

Las variables han sido evaluadas mediante calificaciones de "S" o "N", según esté presente o no el criterio evaluado, tal y como se refleja en la tabla 2 . La calificación " $\mathrm{N}$ " indica que no se ha encontrado esa característica reflejada en el texto.

De los 12 estudios aceptados y evaluados mediante la escala PEDro, 9 presentan 
una buena calidad metodológica ${ }^{15-18,20-23,25}$. A excepción del estudio realizado por Leach W. y col ${ }^{19}$ y Chen LH y col ${ }^{14}$ en el resto de las investigaciones los sujetos fueron asignados al azar (criterio 2) y presentan una buena adhesión de la muestra (criterio 8). En más de la mitad de los trabajos analiza$\operatorname{dos}^{15,16,18,20-22,25}$ la asignación fue realizada de manera oculta (criterio 3).

En tres de los doce estudios ${ }^{20-22}$ los sujetos fueron cegados en la asignación de los grupos (criterio 5). Sin embargo, los terapeutas presentan enmascaramiento solo en $\operatorname{dos}^{20,22}$ (criterios 6). En todas las investigaciones todos los evaluadores que midieron algún resultado clave fueron cegados (criterio 7), salvo los trabajos realizados por Chen y coll ${ }^{14}$, Boese y col ${ }^{25} \mathrm{y} \mathrm{Alkire}^{24}$.

\section{DISCUSIÓN}

En el presente trabajo se han intentado determinar los posibles beneficios que la aplicación de la movilización pasiva continua puede aportar junto con el tratamiento rehabilitador de fisioterapia en el postoperatorio inmediato de pacientes sometidos a una intervención de artroplastia total de rodilla, ya que uno de los factores más importantes que condicionan los beneficios de la sustitución protésica de rodilla es una adecuada atención postoperatoria ${ }^{26}$.

A pesar de que la movilización pasiva continua data de 1980, existen pocos ensayos clínicos en los que se haya investigado sobre los beneficios que aportaría su aplicación. En este aspecto, en esta revisión bibliográfica, solo se han encontrado 12 ensayos clínicos publicados en los últimos 10 años. Debido a esto, quizás sería conveniente incrementar el periodo de búsqueda para determinar más exactamente la eficacia o no de la técnica y su posible recomendación al personal sanitario, especialmente a los fisioterapeutas.

Aunque las investigaciones encontradas sobre la temática son escasas, la mayoría de los estudios que componen esta revisión son de una buena calidad metodológica según la escala PEDro ${ }^{12,13}$, y, además, presentan resultados similares en cuanto al efecto de la aplicación de la movilización pasiva continua junto con el tratamiento de fisioterapia sobre el rango de amplitud articular y sobre el dolor, principalmente.

La deducción principal de esta revisión sistemática sugiere que el uso de la movilización pasiva continua junto con el tratamiento de fisioterapia tras la intervención de artroplastia total de rodilla no es efectivo para aumentar el rango de movimiento articular hacia la flexión activa o pasiva de la rodilla a largo plazo (un año después de la intervención) en comparación con la utilización del tratamiento de fisioterapia solo $^{16,19-22}$. Sin embargo, algunos de los resultados de esta revisión sugieren que la aplicación de la movilización pasiva continua junto con el tratamiento de fisioterapia tras la intervención de rodilla, puede producir pequeños cambios en la amplitud de la flexión de la rodilla a corto plazo (a los 5 y a los 17 días de la intervención) $)^{23,16}$ en comparación con la aplicación de fisioterapia sola.

En cuanto a la amplitud de la extensión activa o pasiva de la rodilla no se obtuvieron resultados estadísticamente significati$\operatorname{vos}^{20,23}$, aunque esta variable fue estudiada por pocos autores ${ }^{19,20,23}$. Sin embargo, en el estudio que realizaron Leach y col $^{19}$ pudieron observar cómo el grupo al que se le aplicó la movilización pasiva continua junto con el tratamiento de fisioterapia, no experimentaba una mayor pérdida de amplitud articular de extensión. No obstante, no es sorprendente que no se valorara en todos los estudios la amplitud articular de la extensión, ya que la máquina de movilización pasiva continua está destinada, principalmente, a mejorar el rango de amplitud articular hacia la flexión. Por lo tanto, se sugiere que, cuando se utilice la movilización pasiva en futuras investigaciones, se incluya un seguimiento en el punto de máxima extensión para activar también el cuádriceps y mejorar la amplitud articular de la extensión activa.

Para algunos autores ${ }^{20,23}$, el uso de la movilización pasiva continua junto con el tratamiento de fisioterapia, sí ofrece resultados estadísticamente significativos en cuanto al dolor postoperatorio, mientras que para otros autores ${ }^{19,21}$ no existieron ta- 
les diferencias. Pensamos que esto puede ser debido al momento en el que cada uno de los autores decidió comenzar a aplicar la técnica. En los estudios de Bruun-Olsen y col' ${ }^{20}$, Bennett y coll ${ }^{23}$ la primera aplicación de movilización pasiva continua se realizó el mismo día de la intervención de la artroplastia total de rodilla y se pudo observar que se mejoraba el dolor en comparación con los pacientes a los que no se les aplicó, mientras que en los dos últimos estudios (Leach y col $^{19}$ y Mainar y col $^{21}$ ) se decidió comenzar al día siguiente y no se obtuvo ninguna mejora en cuanto al dolor. Consideramos pues, que sería necesaria la realización de más investigaciones en las que se establezca comenzar con el uso de la movilización pasiva continúa el mismo día de la intervención de rodilla, para poder obtener resultados más fiables.

Teniendo en cuenta el pensamiento tradicional tanto de profesionales sanitarios, fisioterapeutas, como de los pacientes, de que el dolor de rodilla durante el postoperatorio va asociado a la inflamación de la articulación que ha sido intervenida quirúrgicamente, habría que suponer que si al aplicar la movilización pasiva continua junto con el tratamiento de fisioterapia se reduce el dolor, como se ha explicado anteriormente, también se tendría que producir una disminución significativa de la inflamación de la rodilla correspondiente ${ }^{20}$. Sin embargo, pocos son los trabajos ${ }^{20}$ en los que se valoró la inflamación de la rodilla y se observó que no se produce esa disminución de la inflamación.

Estos resultados se asemejan a los obtenidos en el estudio de Mainar y col $^{21}$, en el que se comprobó que en los grupos en los que se aplicó movilización pasiva continua junto con el tratamiento de fisioterapia tras la intervención de rodilla se retrasaba aún más la disminución de la inflamación de la misma, tardando hasta tres meses en volver a la normalidad, mientras que en el grupo que solamente recibía el tratamiento de fisioterapia postoperatorio, la vuelta a la normalidad de la rodilla se producía a los 42 días de la intervención. En este caso, al igual que en el anterior, creemos que sería aconsejable que en futuras investigaciones se tuviera en cuenta esta medida para poder obtener más resultados, y así poder determinar si resulta o no beneficiosa la movilización pasiva continua en cuanto a la inflamación de la rodilla.

La información obtenida (Test Up and $G^{20,21}$; Curación de las heridas ${ }^{21,23}$; duración de la estancia hospitalaria ${ }^{22,23}$; función de la rodilla ${ }^{23}$; percepción del estado de salud percibido por los pacientes ${ }^{23}$; adherencia de las intervenciones ${ }^{22}$; estado funcional ${ }^{16}$ ) es limitada, ya que la mayoría de las medidas han sido evaluadas en un solo estudio y, por tanto, no da pie a su comparación ni a establecer una conclusión definitiva. Debido a ello, pensamos que sería aconsejable que estas medidas fuesen valoradas en investigaciones futuras y por varios investigadores para poder establecer conclusiones fiables sobre ellas.

Esta heterogeneidad entre las medidas de resultado que se tomaron en cada uno de los diferentes estudios analizados, ha representado un problema a la hora de establecer comparaciones fiables. Otro factor que dificulta la obtención de conclusiones finales es que los protocolos que se aplicaron variaron entre un ensayo y otro. Además de no existir un consenso en cuanto a las características clínicas de aplicación de la movilización pasiva continua, como son la amplitud de movimiento seleccionada para el tratamiento, cuándo comenzar con su aplicación, duración del tratamiento, intensidad de la aplicación, etc.

Según los resultados que se han podido conseguir de esta revisión, la aplicación de movilización pasiva continua junto con el tratamiento de fisioterapia tras la intervención de artroplastia total de rodilla, en comparación con el tratamiento postoperatorio de fisioterapia, no ofrece ningún tipo de beneficio en ninguna de las medidas evaluadas. Aunque, hay que destacar que a corto plazo sí presenta un aumento de la amplitud articular a la flexión, lo cual puede resultar bastante motivante para el paciente y para la continuación de su rehabilitación. En este aspecto nuestros resultados, actualizados en 2014, coinciden con otras revisiones realizadas en años anteriores $\left(2002,2004\right.$ y 2009) ${ }^{27-29}$, aunque se evi- 
dencian resultados favorables a corto plazo en la flexión de rodilla, que en ocasiones disminuyen la estancia de los pacientes en el hospital ${ }^{27}$, no se han encontrado beneficios a largo plazo por lo que se necesita seguir investigando sobre esta temática.

Así pues, y por lo anteriormente dicho, consideramos que sería recomendable la realización de futuras investigaciones, estableciendo primeramente un protocolo internacional de actuación estandarizado, de tal manera que todas las investigaciones que lleguen a realizarse tengan un mínimo de parámetros coincidentes, como puedan ser el número mínimo de pacientes estudiados, el momento de inicio de aplicación de la técnica, las sesiones al día y el tiempo de cada sesión, periodos de toma de resultados o medidas de resultado mínimas a valora.

\section{BIBLIOGRAFÍA}

1. RiBERA JM. Epidemiología de la enfermedad osteoarticular en la persona mayor. JANO 2003; 64: 21-27.

2. López-Liria R, Vega-Ramirez FA, López-Liria R, Vega-Ramírez FA, Catalán-Matamoros D, PadillaGóngora D, Martínez-Cortés MC, Mesa-Ruiz A. La rehabilitación y fisioterapia domiciliaria en las prótesis de rodilla. An Sist Sanit Navar 2012; 35: 99-113.

3. Gómez PAvón J. Calidad de vida relacionada con la salud. La punta del iceberg del complejo abordaje de los ancianos muy mayores. Med Clin 2010; 135: 162-164.

4. Batlle-Gualda E. Estudio ArtRoCad: evaluación de la utilización de los recursos sanitarios y la repercusión socioeconómica de la artrosis de rodilla y cadera. Presentación de resultados preliminares. Rev Esp Reumatol 2005; 32: 22-27.

5. Frías G, Castro MC, Caracuel ma, Collantes E. Abordaje terapéutico de la artrosis. JANO 2011: 1771: 55-60.

6. Bernard Pineda M. Actualización en artrosis. Comisión de formación continuada. Sistema Nacional de Salud. Grupo SANED 2007.

7. Hunter DJ, Lo, GH. The management of osteoarthritis: an overview and call to appropiate conservative treatment. Rheum Dis Clin North Am 2008; 34: 689-712.

8. Jurado-Moyano M. Pronóstico en la evolución de los pacientes con prótesis total de rodilla. Estudio de una serie de casos. Cuestiones de fisioterapia 2011, 40: 204-210.
9. Cruz Salinas AL, Guevara Ortega MI, Herazo Mutis DJ, Tapasco Gutiérrez GA. Valoración biomecánica de la unicac de movimiento pasivo continuo para rodilla, fabricada en el programa de ingeniería mecánica de la Universidad del Valle, al ser aplicada en personas sanas [Trabajo de Grado]. Santiago de Cali: Universidad del Valle. 2005.

10. León JC, Gálvez DM, Arcas MA, Paniagua SL, PeLLICER M. Fisioterapeutas del Servicio Gallego de Salud. Vol 2, 1를. Sevilla: MAD; 2006.

11. Saló JC, Cardona JM, SÁnchez M, Orán J, ForCADA P, FERNÁNDEZ JJ. Valoración de los beneficios de la movilización pasiva tras artroplastia total de rodilla. Rev Esp Cir Osteoartic 2002; 37: 121-124.

12. Escala P. [Consultado 18/12/2014]. Disponible en: http://www.pedro.org.au/spanish/ downloads/pedro-scale/.

13. Moseley AM, Herbert RD, Sherrington C, Maher CG. Evidence for physiotherapy practice: a survey of the Physiotherapy Evidence Database (PEDro). Aust J of Physiother 2002; 48: 43-49.

14. Chen LH, Chen CH, Lin SY, Chien SH, Su JY, HuANG CY et al. Aggressive continuous passive motion exercise does not improve knee range of motion after total knee arthroplasty. J Clin Nurs 2013; 22: 389-394.

15. Mau-Moeller A, Behrens M, Finze S, Bruhn S, Bader R, Mittelmeier W. The effect of continuous passive motion and sling exercise training on clinical and functional outcomes following total knee arthroplasty: a randomized active-controlled clinical study. Health Qual Life Outcomes 2014; 12: 68.

16. Lenssen TA, VAn Steyn MJ, CRiJns YH, Waltjé EM, Roox GM, GEesink RJ et al. Effectiveness of prolonged use of continuous passive motion (CPM), as an adjunct to physiotherapy, after total knee arthroplasty. BMC Musculoskelet Disord 2008; 9: 60 .

17. Herbold Ja, Bonistall K, Blackburn M, Agolli J, Gaston S, Gross C et al. Randomized controlled trial of the effectiveness of continuous passive motion after total knee replacement. Arch Phys Med Rehabil 2014; 95: 1240-1245.

18. Davies DM, Johnston DW, Beaupre LA, LiER DA. Effect of adjunctive range-of-motion therapy after primary total knee arthroplasty on the use of health services after hospital discharge. Can J Surg 2003; 46: 30-36.

19. Leach W, Reid J, Murphy F. Continuous passive motion following total knee replacement: a prospective randomized trial with follow-up to 1 year. Knee Surg Sports Traumatol Arthrosc 2006; 14: 922-926. 
20. Bruun-Olsen V, Heiberg Ke, Mengshoel AM. Continuous passive motion as an adjunct to active exercises in early rehabilitation following total knee arthroplasty: a randomized controlled trial. Disabil Rehabil 2009; 31: 277283.

21. Mainar RN, Baviskar JV, Singhi T, Rathi SS. To use or not to use continuous passive motion post-total knee arthroplasty. Presenting functional assessment results in early recovery. J Arthroplasty 2012; 27: 193-200.

22. Denis M, Moffet H, Caron F, Ouellet D, Paquet J, Nolet L. Effectiveness of continuous passive motion and conventional physical therapy after total knee arthroplasty: a randomized clinical trial. Phys Ther 2006; 86: 174-185.

23. Bennett LA, Brearley SC, Hart JA, Bailey MJ. A comparison of 2 continuous passive motion protocols after total knee arthroplasty. A controlled and randomized study. J Arthroplasty 2005; 20: 225-233.

24. AlkiRe MR, Swank ML. Use of inpatient continuous passive motion versus no $\mathrm{CPM}$ in computer-assisted total knee arthroplasty. Orthop Nurs 2010; 29: 36-40.

25. Boese CK, Weis M, Phillips T, Lawton S, Gallo T, CEnteno L. The efficacy of continuous passive motion after total knee arthroplasty: a comparison of three protocols. J Arthroplasty 2014; 29: 1158-1162.

26. BRANDI DE LA ToRre, JM. Evaluación de la intervención de fisioterapia en pacientes portadores de una artroplastia total de rodilla. Un ciclo de calidad [Máter Universitario]. Universidad de Murcia. Departamento de Ciencias Sociosanitarias, Facultad de Medicina. Julio 2012.

27. Milne S, Brosseau L, Robinson V, Noel MJ, Davis $\mathrm{J}$, Droyin $\mathrm{H}$ et al. Continuos passive motion following total Knee arthroplasty. Cochrane Database Syst Rev 2003; (2): CD004260.

28. Brosseau L, Milne S, Wells G, Tugwell P, RobinSON V, CASIMIRo L et al. Efficacy of continuous passive motion following total knee arthroplasty: a metaanalysis. J Rheumatol 2004; 31 : 2251-2264. 